\title{
Blade cutting of thin walled structures by explicit dynamics finite elements
}

\author{
Federica Confalonieri · Aldo Ghisi · Umberto Perego
}

Received: date / Accepted: date

\begin{abstract}
In cohesive crack propagation induced by blade cutting, it is necessary to consider the blade radius of curvature as a characteristic length additional to the shell thickness and to the cohesive process zone length, which usually characterize crack propagation in thin walled structures. When the finite element simulation of a blade cutting process is considered, these three lengths need to be properly resolved. The blade radius of curvature can be orders of magnitude smaller than the shell thickness and the cohesive process zone. Furthermore, the transition from a continuous mesh to a mesh containing a crack with a cohesive interface is well known to be critical for solution accuracy. Nodal equilibrium is in general violated during the transition, with subsequent generation of spurious stress oscillations that, in view of the non-reversible nature of the problem, can lead to significant inaccuracies in the stress response. The smallest length, i.e. the blade radius of curvature, is here resolved using the so called directional cohesive element model as in Pagani and Perego (CMAME, 285, 515-541, 2015), while the structural thickness is modeled using solid-shell elements. The concept of directional cohesive elements is here extended for application to the case of cutting by scissors. As for the cohesive process zone length, different modeling options are discussed in terms of their capability to reduce the spurious oscillations and to provide an accurate estimate of the cutting parameters. Numerical tests are presented to validate the proposed modeling strategies.
\end{abstract}

F. Confalonieri E-mail: federica.confalonieri@polimi.it

. A. Ghisi E-mail: aldo.ghisi@polimi.it

· U. Perego E-mail: umberto.perego@polimi.it

Department of Civil and Environmental Engineering, Politecnico di Milano, piazza L. da Vinci, 32, Milano (Italy)
Keywords Blade Cutting · Explicit Dynamics · Cohesive Crack Propagation · Spurious Stress Oscillations . Directional Cohesive Elements

\section{Introduction}

The simulation of crack propagation in shell structures is a complex problem, still attracting considerable attention in the computational mechanics community. Among the most common approaches, one can list the element deletion technique, whereby an element is eliminated from the mesh when a fracture criterion is satisfied at that element (see e.g. Ortiz and Pandolfi [23]), smeared approaches, such as those based on the phase field method (see e.g. Ulmer et al. [36], Amiri et al. [4], Ambati and De Lorenzis [3]), and techniques based on the explicit introduction of a displacement discontinuity in the finite element displacement model. This can be done by either enriching the displacement field, as e.g. in the extended finite element method (XFEM, see e.g. Dolbow et al. [10] and Areias and Belytschko [5]), or by allowing a crack to propagate along element edges whose nodes have been duplicated (see e.g. Cirak et al. [8] and Pagani and Perego [22]). In this latter case, a node is duplicated when a fracture criterion is satisfied at its position and a crack is let to propagate by separating adjacent elements along the previously common edge starting from that node. Cohesive interface elements are usually interposed between the separating shell element faces, to account for the progressive release of fracture energy. In blade cutting problems, the main crack propagation direction is dictated by the imposed blade trajectory, so that the mesh can be designed to follow the main propagation path, which is known in advance. For this reason, the node separa- 
tion method can be conveniently adopted for this type of problems, without the need to use extremely refined meshes. In view of the high nonlinearity of the problem, the finite element simulation of cutting is here dealt with in an explicit dynamics framework.

In the case of cohesive crack propagation through thin shells promoted by blade cutting, the problem is dominated by the existence of three small geometric scales. The first one is the scale of the thickness of the thin-walled structure, which is usually orders of magnitude smaller than the in-plane global dimensions. The second small scale is dictated by the size of the cohesive process zone and depends on the used material and on the problem geometry. The smallest scale is determined by the curvature radius of the cutting blade, which can be of the order of microns, or even less, in the case of a sharp blade. While the first scale is usually resolved using shell elements, the resolution of the second scale often requires extremely fine meshes, with high computational costs. As for the third scale, for sharp blades and ductile materials the cutting blade is likely to interfere with the correct transmission of the cohesive tractions between the two separating crack flanks, leading to possible severe underestimation of the dissipated energy [22], and needs a special treatment. Unfortunately, the blade curvature radius turns out to be by far smaller than the typical size of a computationally acceptable in-plane discretization of the process zone, so that the interaction cannot be properly resolved.

In the present work, the problem of the blade sharpness is addressed by means of the Directional Cohesive Elements (DCEs) introduced in Frangi et al. [11] and further developed in Pagani and Perego [22] and Confalonieri et al. for layered shells [12]. According to this technique, when a crack propagation criterion is met at a node, the node is duplicated and cohesive directional elements, i.e. a sort of cohesive string elements, which have the purpose to simulate the interaction between the blade and the deforming material in the process zone and are endowed with a proper amount of cohesive energy, are attached to the opening faces. During the process of blade cutting, the sharp blade interacts with the material in the deforming process zone and complex nonlinear deformations take place ahead of the crack tip. According to the cohesive model of fracture, the three-dimensional process zone ahead of the crack tip is collapsed onto a two-dimensional zero-thickness interface. Upon interface opening, cohesive tractions are transmitted between the separating crack flanks. From the geometrical point of view, however, the interface opening creates an empty space between the separating crack flanks that does not exist in reality, since that space is occupied by the material in the deforming process zone. When the crack is produced by a sharp cutting blade, the blade can enter the space fictitiously created by the opening of the cohesive interface, without any interaction with the material in the process zone. As shown in quantitative terms in Pagani and Perego (2015) [22], this lack of interaction may lead to a severe underestimation of the dissipated energy, unrealistically postponing crack propagation. The DCEs are a sort of cohesive string elements, which have the purpose to account for the interaction between the blade and the deforming material in the process zone and are endowed with a proper amount of cohesive energy. In contrast to classical cohesive elements, the DCEs are geometric entities and are able to detect contact with the cutting blade. Because of contact with the blade, the DCEs deform, transmitting cohesive forces to the two crack flanks in the correct directions. The correct amount of cohesive energy is then dissipated through the string elongation (see figure 1), until the final rupture of the string element is achieved. In the particular case of cutting by scissors, the interaction between the scissors blades has to be taken into account. Using simple arguments, the concept of directional cohesive elements is here extended to include also the case of cutting scissors.

Despite the improved energy balance allowed by the DCEs in the presence of a cutting blade, crack propagation in explicit dynamics is often accompanied by significant spurious stress oscillations. The first reason is that the cohesive characteristic length needs to be properly resolved with a sufficiently fine discretization to accurately capture the cohesive stress distribution. This requires the size of the cohesive zone to be estimated for mesh design. When this zone is very small compared to the structure size, the cohesive length turns out to depend on material properties (Young modulus, peak strength, toughness and adopted cohesive law) and on the fracture mode. In the case of slender structures, as in the case of laminates, the structure geometry (e.g. the plies thickness) also plays a role. Several contributions are available in the literature with analytical estimates of the size of the cohesive process zone (see Soto et al. [31] for a recent review of the available estimates). However, numerical verifications (see e.g. Harper and Hallett [14] and Turon et al. [33]) have shown that these analytical predictions overestimate the actual cohesive length in most cases. A reduction factor of one half is recommended in [14], while Soto et al. [31] propose new empirical formulas to predict the cohesive zone length in pure Mode I or Mode II. Once the cohesive length has been estimated, the mesh has to be designed in such a way that a sufficient number of finite elements is used along its length. 


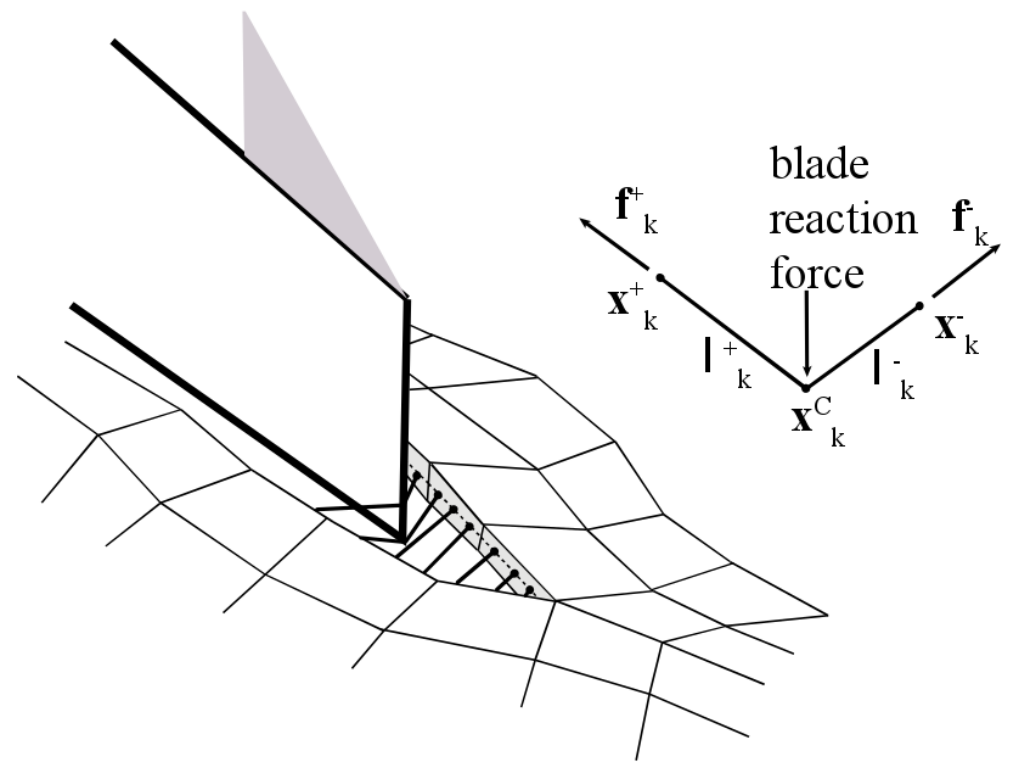

Fig. 1 Distribution of cohesive forces via the "directional cohesive element" concept.

A second source of spurious stress oscillations is due to the fact that, when a node is duplicated to allow for crack propagation, at the instant of the (either directional or classical) insertion of the cohesive element, the nodal force balance may not be satisfied, since the cohesive tractions applied to the element side in general do not match the nodal forces that were transmitted by the elements sharing that node before it was duplicated.

These problems, which are present also with standard cohesive elements, are well known and have been discussed extensively in the literature on finite element cohesive models. Concerning the size of the cohesive process zone, the specific shape of the adopted tractiondisplacement curve has been shown to have a significant effect on the computed cohesive zone length and on the traction distribution along the cohesive zone (see e.g. Planas and Elices [24], Smith [30], Volokh [37], Shet and Chandra [28], Alfano [1], Harper and Hallet [14]). However, when the geometric length scale of interest is sufficiently large relative to the cohesive length scale (Needleman [21]), and when only the global loaddisplacement structural response is of interest, details of the cohesive law play a minor role and the results are only slightly affected by the exact shape of the cohesive curve provided that the material fracture energy is correctly accounted for (Yang and Cox [38]). Based on this consideration, it is possible to adjust the cohesive strength, which is anyway difficult to measure experimentally, and the critical opening with the purpose to increase the cohesive zone length and to relax the constraint on the mesh density in the process zone. In particular, it has been shown (Planas and Elices [24], Smith
[30]) that increasing the critical opening displacement at constant toughness (at the cost of a reduction of the cohesive strength) can lead to a significant increase in the cohesive length. The adaptive definition of these latter parameters has also been proposed as a systematic technique to improve accuracy in the presence of coarse finite element discretizations of the process zone (Turon et al. [34,32]). A similar technique has also been used by $\mathrm{Hu}$ et al. [15], who gradually reduced the peak stress and the initial stiffness of the cohesive law in the pre-softening regime, depending on the effective opening displacement. The approach is shown to produce more accurate results with a lower computational cost. However, recent studies (Blackman et al., Harper and Hallet) have shown that excessively low values of cohesive peak strength can lead to inaccurate results.

Another possible approach to reduce stress oscillations, consists of improving the integration rule, so as to obtain a more accurate description of the stress distribution along the crack edge, without increasing the number of finite elements in the process zone. Shellekens and De Borst [26] found that the Newton-Cotes integration (NCI) rule performed better than Gauss Integration (GI) for both linear and quadratic cohesive elements. In the latter case, they ascribed the merit to the integration point located at the face centroid. Alfano and Crisfield [2], however, showed that a higher order of the numerical integration is not sufficient to allow for a coarser mesh discretization. Recently, Gilormini and Diani [13] discussed the effect of the numerical integration rule in the case of finite strains. Their numerical peeling experiment showed no sensible differences be- 
tween NCI and GI rules in nonlinear regimes, while a less precise result has been observed for NCI in the elastic part of the cohesive law. The possibility to exploit numerical integration to improve the cohesive element computational efficiency has been investigated also by Yang et al. [39], who introduced a cohesive element featuring a multiple subdomain integration: the numerical integration for each cohesive finite element is subdivided in zones, whose dimensions are smaller than the cohesive zone size, inside which GI or NCI schemes are employed. This approach makes it possible to increase the mesh size from a fraction (typically $\frac{1}{3} \div \frac{1}{5}$ ) of the cohesive zone to a size comparable and even slightly larger of the cohesive zone. Moreover, the modifications in a standard finite element implementation are quite limited, see [25].

To mitigate the oscillations due to unbalanced nodal forces upon the insertion of a new cohesive segment in an extended finite elements implementation (XFEM), Menouillard and Belytschko $[18,19]$ proposed to add an artificial correction of nodal forces. In their correction method, fictitious forces are added to the interested nodes to re-establish nodal equilibrium. These forces are then progressively scaled down when the crack tip reaches the next element edge in the propagation path. The same technique has been used, e.g., by Mostofizadeh et al. [20] for the simulation of dynamic crack propagation in thin shells.

In view of these considerations, a correction similar to the one proposed in $[18,19]$ is considered in this work. The required correction force is calculated at the node in correspondence of the newly separating element crack tip, so as to compensate the missing force contribution coming from the adjacent element before separation. The additional contribution is however provided by a modification of the maximum traction and critical opening in the cohesive law, while preserving the cohesive energy. It should be emphasized that a numerical manipulation of the cohesive behavior of this kind is acceptable only when global responses are of primary concern, since the modification will obviously affect the local response in the crack tip region.

The correction has been implemented in an explicit dynamics solid-shell finite element code together with the DCE technique. The effect on accuracy of the number of introduced string elements and of the proposed correction is investigated by application to test cases taken from the literature. In the next section 2 the basics of the DCEs, the rules for the numerical integration in the case of multiple DCEs along an element face and the adopted correction scheme are briefly summarized, while validation tests are presented in section 3 . Con- clusions are drawn in section 4 . In all the paper, bold characters are reserved for vectors or matrices.

\section{Formulation}

\subsection{Governing equations}

Let us consider a thin-walled structure, modelled as a deformable body undergoing large deformations. The deformation mapping $\chi(\mathbf{X}, t), t \in[0, T]$ (being $\mathbf{X}$ the initial position vector and $T$ the overall analysis time) is introduced to describe the motion of the body from its initial configuration $B_{0}$, characterized by volume $\Omega_{0}$ with boundary $\partial \Omega_{0}$, to the current one $B$, in which it occupies the volume $\Omega$ with boundary $\partial \Omega$. The body is crossed by a cohesive crack propagating through its thickness: because of the deformation process, the zero thickness interface $\Gamma_{0}$ in the reference configuration transforms into the interface $\Gamma$ in the current configuration. The discontinuity across the interface $\Gamma$ is described by the relative displacement vector, defined as $\boldsymbol{\delta}=\mathbf{x}^{+}-\mathbf{x}^{-}$, being $\mathbf{x}^{+}$and $\mathbf{x}^{-}$the spatial coordinates of two corresponding points belonging to the separating flanks of the cracks, denoted as $\Gamma^{ \pm}$respectively.

In the absence of damping, the dynamical behaviour of the deformable body can be described by means of the following variational balance:

$\delta \Pi_{\mathrm{kin}}^{e}+\delta \Pi_{\mathrm{int}}^{e}-\delta \Pi_{\mathrm{ext}}^{e}=0$

where $\Pi_{\mathrm{kin}}^{e}$ is the kinetic energy, while $\Pi_{\mathrm{int}}^{e}$ and $\Pi_{\mathrm{ext}}^{e}$ are the internal and external works respectively. The kinetic contribution is expressed as:

$\delta \Pi_{\mathrm{kin}}^{e}=\left[\int_{\Omega} \rho \ddot{\mathbf{u}} \cdot \delta \mathbf{u} \mathrm{d} \Omega\right]$

being $\rho$ the mass density, $\mathbf{u}$ the nodal displacements vector and $\ddot{\mathbf{u}}$ the nodal accelerations vector. The internal work variation is given by two distinct contributions $\delta \Pi_{\mathrm{B}}^{e}$ and $\delta \Pi_{\mathrm{C}}^{e}$, related to the bulk material and to the cohesive interface respectively, i.e.

$\delta \Pi_{\mathrm{int}}^{e}=\delta \Pi_{\mathrm{B}}^{e}+\delta \Pi_{\mathrm{C}}^{e}$

with:

$\delta \Pi_{\mathrm{B}}^{e}=\int_{\Omega_{0} \backslash \Gamma_{0}} \mathbb{S}: \delta \mathbb{E} \mathrm{d} \Omega_{0}$

$\delta \Pi_{\mathrm{C}}^{e}=\int_{\Gamma} \mathbf{T} \cdot \delta \boldsymbol{\delta} \mathrm{d} \Gamma$ 
where $\mathbb{S}$ is the second Piola-Kirchhoff stress tensor, $\mathbb{E}$ the Green-Lagrange strain tensor, $\mathbf{T}$ the Cauchy traction vector in the current configuration, defined as:

$$
\mathbf{T}=\boldsymbol{\sigma} \mathbf{n}
$$

being $\boldsymbol{\sigma}$ the Cauchy stress tensor and $\mathbf{n}$ the normal to the crack flank.

Starting from the virtual balance of eqn. 1, the following set of semi-discretized global equations can be written for the deformable body as:

$\mathbf{M U ̈}+\mathbf{F}_{i n t}+\mathbf{F}_{c o h}-\mathbf{F}_{e x t}=\mathbf{0}$

being $\mathbf{M}$ the mass matrix, $\ddot{\mathbf{U}}$ the global acceleration vector, $\mathbf{F}_{\text {int }}$ the vector of internal forces due to the bulk contribution, $\mathbf{F}_{c o h}$ the vector of cohesive forces and $\mathbf{F}_{e x t}$ the external load vector.

As in [22], the deformable body is discretized by means of solid-shell elements, while the blade is modeled as a rigid body, whose surface is meshed with triangular elements. The solid-shell formulation developed in [27] has been adopted. This 8-node element makes use of 24 displacement degrees of freedom, one integration point in the in-plane direction, with five integrations points along the thickness, and its formulation is based on reduced integration with hourglass stabilization, enhanced assumed strain (EAS) and assumed natural strain (ANS) to prevent locking. Figure 2 depicts a generic solid-shell element, i.e. a 8-node brick, whose node numbering is sorted so that nodes 1-4 belong to the lower surface, while nodes 5-8 are on the upper surface. The identification of the brick's lower and upper surface is straightforward, given the small thickness of the element. In the following, the nodes of the solidshell elements will be denoted as vertex nodes. Let us define the corner fiber as the edge connecting two corresponding vertex nodes belonging to the lower and upper surfaces, respectively, and the corner nodes as the middle surface nodes, i.e. the midpoints of each of the four corner fibers. The coordinates of the corner nodes in the undeformed configuration and their displacements are defined as:

$$
\begin{aligned}
\mathbf{X}_{i}^{m} & =\frac{\mathbf{X}_{i}^{u p}+\mathbf{X}_{i}^{\text {low }}}{2} \\
\mathbf{u}_{i}^{m} & =\frac{\mathbf{u}_{i}^{\text {up }}+\mathbf{u}_{i}^{\text {low }}}{2}
\end{aligned}
$$

where the subscript $m$ refers to the corner node, while the superscripts up and low denote quantities related to the vertex nodes sharing the same fiber and belonging to the upper and lower surfaces respectively. $\mathbf{X}_{i}^{m, u p, l o w}$ and $\mathbf{u}_{i}^{\text {m,up,low }}$ are the vectors collecting the material coordinates and the displacements of the nodes, being $i$ the corner fiber number.

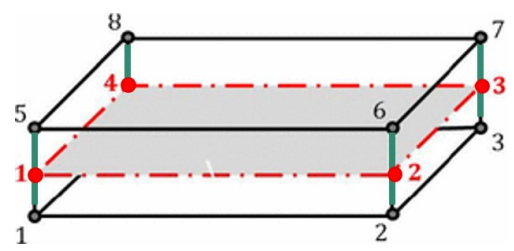

Fig. 2 8-node solid-shell element. Black circles: vertex nodes; red circles: corner nodes; red dashed lines: edges of the element middle surface; vertical dark green lines: corner fibers.

\subsection{Directional Cohesive Elements (DCEs)}

The fracture propagation is handled making use of an inter-element cohesive approach, justified by the presence of a dominant crack path driven by the blade trajectory: the interface elements are interposed on the fly between two adjacent, previously connected solid-shell elements, where an activation test is satisfied. The Directional Cohesive Elements (DCEs) proposed in [11, 22], i.e. massless string elements able to detect contact with the blade on the basis of purely geometric considerations, are adopted to properly describe the interaction between the blade and the cohesive process zone.

The activation test is performed considering principal stresses or strains at the corner nodes, depending on the adopted bulk constitutive behaviour. If the activation criterion is satisfied at a corner node, the corresponding corner fiber is duplicated and a new face is created. In [11] and [22] only one DCE per fiber connecting the pair of separating corner nodes was introduced. In this work, an increased number of DCEs per element face is considered to provide a better representation of the cutting forces when the blade crosses the cohesive process zone. The cohesive strings are attached to the Gauss points lying on the crack faces, at the level of the solid-shell element middle surface (i.e. only one DCE is inserted through the thickness in view of the small thickness of the body), as shown in Figure 3. Since the solid-shell elements are always thin compared to the in-plane dimensions, the energy dissipated (in the absence of contact with the blade) with one or more DCEs along the face thickness is almost the same. A better resolution of crack propagation along the thickness can be achieved by using more solid-shell elements in thickness direction, each with one DCE along the thickness, as discussed in [12] for layered shells.

As long as there is no contact with the blade, the DCE works as a classical cohesive element, connecting along a straight line two originally coincident Gauss points, so that the string length measures their relative displacement. The cohesive forces $\mathbf{T}^{+}$and $\mathbf{T}^{-}$are transmitted along the direction of the string and their magnitude $T$ depends on the adopted cohesive model. 
Consequently, the DCE provides co-linear relative displacements and tractions.

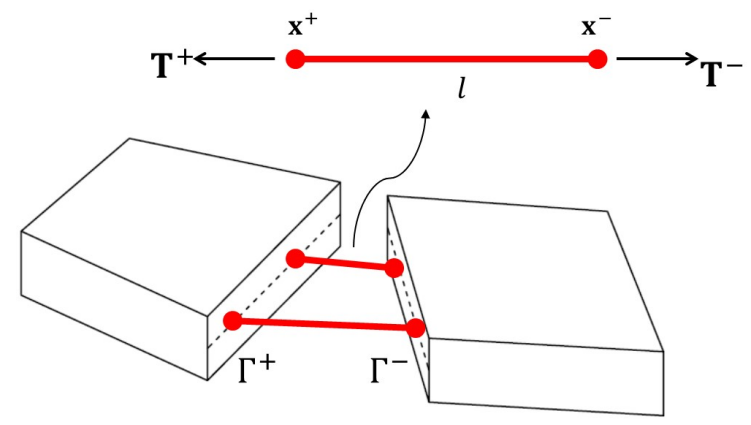

Fig. 3 Directional cohesive elements attached to points lying on the elements middle surfaces.

Once contact with the blade is detected, the original cable element is subdivided into two branches separated by the contact point, as shown in Figure 4. This point is assumed to remain in contact with the same element of the blade throughout all the analysis, under the hypothesis of high friction. The directions of the two cohesive traction vectors are determined by the inclinations of the two branches of the string element and, thus, are influenced by the position of the contact point. The tractions $\mathbf{T}^{+}$and $\mathbf{T}^{-}$transmitted by the string branches to the crack flanks are assumed to have the same magnitude $T$. This depends on the total string length $l$, which plays the role of an effective interface opening, different from the relative displacement, and is given by the sum of the lengths of the two branches of the string $l=l^{+}+l^{-}$.

In this work, the magnitude of the cohesive force transmitted by the string element at the two opening faces is ruled by the linear softening law depicted in Figure 5, although any other functional form of the softening branch is feasible. The cohesive traction $T$ is a function of the overall cable length $l$ and of the maximum elongation $\bar{l}$, which works as the historical variable and allows to distinguish between the loading and the unloading phases. The cohesive law is described by the following set of equations:

$$
\begin{array}{cll}
T=T_{0} \frac{l_{c}-l}{l_{c}} & \text { for } l \geq \bar{l} & \text { loading } \\
T=T_{0} \frac{l_{c}-\bar{l}}{l_{c}} \frac{l}{l} & \text { for } l<\bar{l} & \text { unloading } \\
T=0 \quad \text { for } l \geq l_{c} & \text { complete decohesion }
\end{array}
$$

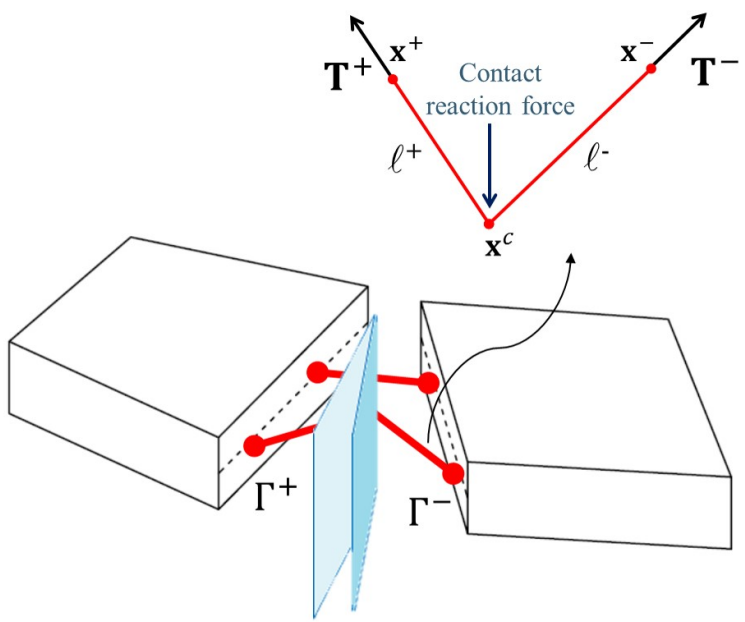

Fig. 4 Directional cohesive element after contact detection.

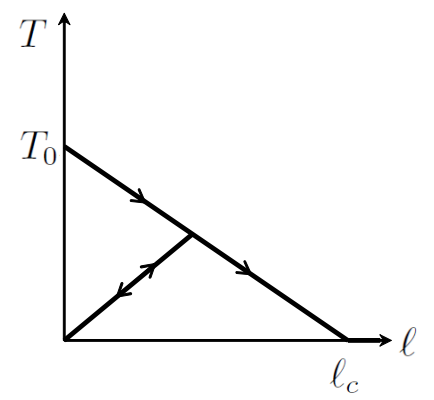

Fig. 5 Linear softening law adopted for the DCE.

being $T_{0}$ the peak cohesive traction and $l_{c}$ the critical cable length corresponding to the ultimate opening. The area beneath the curve represents the fracture energy $\mathcal{G}_{c}=\frac{1}{2} T_{0} l_{c}$. The cohesive tractions transmitted to the crack flanks by a single DCE are, thus, given by:

$\mathbf{T}^{+}=T \mathbf{m}^{+} \quad \mathbf{T}^{-}=T \mathbf{m}^{-}$

If there is no contact between the DCE and the blade, the unit vectors $\mathbf{m}^{+}$and $\mathbf{m}^{-}$are defined as:

$\mathbf{m}^{+}=-\mathbf{m}^{-}=\frac{\mathbf{x}^{+}-\mathbf{x}^{-}}{\left\|\mathbf{x}^{+}-\mathbf{x}^{-}\right\|}=\frac{l}{\left\|\mathbf{x}^{+}-\mathbf{x}^{-}\right\|}$

being $\mathbf{x}^{+}$and $\mathbf{x}^{-}$the spatial coordinates of the two string nodes attached to the crack flanks $\Gamma^{+}$and $\Gamma^{-}$ respectively. In the case of contact between the DCE and the blade, the unit vectors $\mathbf{m}^{+}$and $\mathbf{m}^{-}$are defined by:

$$
\begin{aligned}
& \mathbf{m}^{+}=\frac{\mathbf{x}^{c}-\mathbf{x}^{+}}{\left\|\mathbf{x}^{c}-\mathbf{x}^{+}\right\|}=\frac{l^{+}}{\left\|\mathbf{x}^{c}-\mathbf{x}^{+}\right\|} \\
& \mathbf{m}^{-}=\frac{\mathbf{x}^{c}-\mathbf{x}^{-}}{\left\|\mathbf{x}^{c}-\mathbf{x}^{-}\right\|}=\frac{l^{-}}{\left\|\mathbf{x}^{c}-\mathbf{x}^{-}\right\|}
\end{aligned}
$$


being $\mathbf{x}^{c}$ the coordinates in the current configuration of the contact point between the string and the blade.

Let us now focus on a single 4-node interface element $e$, with an intrinsic reference system $\theta-\zeta$, coinciding with the solid-shell face on the crack flank, as shown in Figure 6. More precisely, the intrinsic coordinates $\theta$ and $\zeta$ are mapped onto the middle plane and the thickness directions of the physical interface element, respectively. The relative displacement model is given by:

$\boldsymbol{\delta}=\left[\sum_{j=1}^{4} N_{j}(\theta, \zeta) \boldsymbol{\delta}_{j}\right]^{e}$

where $N_{j}(\theta, \zeta)$ and $\boldsymbol{\delta}_{j}$ represent the shape function and the relative displacement vector of node $j$. By substituting eqn. 17 into eqn. 5, the elemental cohesive contribution $\delta \Pi_{\mathrm{C}}^{e}$ can be written as:

$\delta \Pi_{\mathrm{C}}^{e}=\int_{-1}^{+1} \int_{-1}^{+1} \mathbf{T}^{e} \cdot \delta\left[\sum_{j=1}^{4} N_{j}(\theta, \zeta) \boldsymbol{\delta}_{j}\right]^{e} J_{\Gamma}^{e} \mathrm{~d} \theta \mathrm{d} \zeta$

being $\mathbf{T}^{e}$ the elemental cohesive traction vector and $J_{\Gamma}^{e}$ the mapping jacobian.

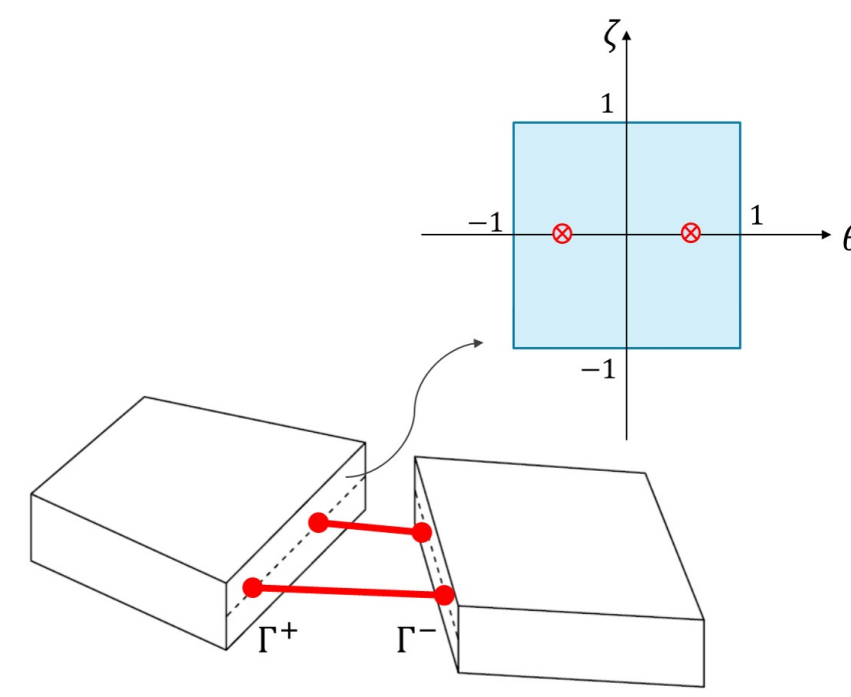

Fig. 6 Master element reference system for the DCEs inserted at the opening surfaces.

A Gaussian quadrature rule with a varying number of Gauss points over $\theta$, placed at $\zeta=0$, is considered (see Table 1). As an example, the case of two Gauss points is depicted in Figure 6. By introducing the integration scheme in eqn. 18, one has:

$\delta \Pi_{C}^{e}=\sum_{j=1}^{4} \sum_{k=1}^{N_{G P}} 2 w_{k} J_{\Gamma_{k}}^{e} \mathbf{T}_{k}^{e} N_{j}\left(\theta_{k}, \zeta=0\right) \cdot \delta \boldsymbol{\delta}_{j}^{e}$
Table 1 Details of the adopted Gaussian integration rule: $N_{G P}$ is the number of Gauss points; $\theta_{k}, k=1, N_{G P}$ are their $\theta$ coordinates in the local reference system; $w_{k}$ are the weights.

\begin{tabular}{ccc}
\hline$N_{G P}$ & $\theta_{k}$ & $w_{k}$ \\
\hline 1 & 0 & 2 \\
2 & \pm 0.577350269 & 1,1 \\
3 & $\pm 0.774596669,0$ & $5 / 9,8 / 9,5 / 9$
\end{tabular}

being $\mathbf{T}_{k}^{e}$ the contribution of the $k$-th DCE to the overall cohesive traction, $N_{G P}$ the total number of Gauss points (coinciding with the number of DCEs per element $),\left(\theta_{k}, \zeta=0\right)$ the local coordinates of the $k$-th integration point and $w_{k}$ its weight. The coefficient 2 that multiplies $w_{k}$ is the weight of the one-point integration in direction $\zeta$.

Upon integration, the elemental cohesive contribution is given by:

$\delta \Pi_{C}^{e}=\sum_{j=1}^{4} \mathbf{f}_{j}^{e} \cdot \delta \boldsymbol{\delta}_{j}^{e}$

being

$\mathbf{f}_{j}^{e}=\sum_{k=1}^{N_{G P}} 2 w_{k} J_{\Gamma_{k}}^{e} \mathbf{T}_{k}^{e} N_{j_{k}}$

the equivalent cohesive force of the interface element $e$ $\theta$ at node $j$ in the global reference frame. Assembling the nodal contributions of the interface elements sharing node $i$, the global cohesive force vector $\mathbf{F}_{c o h, i}$ at node $i$ can be computed as:

$\mathbf{F}_{c o h, i}=\mathcal{A}_{e=1}^{N_{i}} \mathbf{f}_{i}^{e}$

being $\mathcal{A}$ the assembly operator and $N_{i}$ the number of interface elements in the node support.

\subsection{Spurious stress oscillations control}

When a corner fiber is duplicated, the same coordinates, displacements, velocities and accelerations of the original vertex nodes are assigned to the newly created ones. Consequently, at the time instant of the interface element insertion $t_{n d}$, the initial length of the newly inserted DCEs is exactly zero and the nodal cohesive forces are proportional to the peak value of the cohesive traction $T_{0}$ and aligned with the local normal to the crack flanks. The vectors of equivalent nodal forces at the corner node $i$, according to the definition of the 
corner node displacements in eqn. 9 are given by:

$\mathbf{f}_{c o h, i}^{m}=\frac{\mathbf{F}_{c o h, i}^{u p}+\mathbf{F}_{c o h, i}^{l o w}}{2}$

$\mathbf{f}_{i n t, i}^{m}=\frac{\mathbf{F}_{i n t, i}^{u p}+\mathbf{F}_{i n t, i}^{l o w}}{2}$

$\mathbf{f}_{e x t, i}^{m}=\frac{\mathbf{F}_{e x t, i}^{u p}+\mathbf{F}_{e x t, i}^{l o w}}{2}$

$\mathbf{f}_{i n, i}^{m}=\frac{\mathbf{F}_{i n, i}^{u p}+\mathbf{F}_{i n, i}^{\text {low }}}{2}$

where the subscripts coh, int, ext and in identify the cohesive, internal, external and inertia forces respectively. When a corner node is duplicated, the nodal forces are released because of the separation of two previously adjacent elements. As noticed also in $[18,19]$, the cohesive forces transmitted by the newly created interface elements are, in general, not able to restore the force balance. As a consequence of this lack of nodal equilibrium, a spurious stress wave starts to propagate, affecting the numerical solution. Noting that, in the present case of a solid-shell discretization, the equivalent nodal internal force vector consists of the sum of a stabilization term $\mathbf{f}_{i n t, i}^{h g}$, introduced to avoid the development of hourglass modes [27], and a physically relevant term $\mathbf{f}_{i n t, i}^{s}$ deriving from the element tangent stiffness, a two-step nodal correction procedure is introduced to reduce these spurious stress oscillations.

Nodal equilibrium is first imposed at the time $t_{n d}$ of node duplication, considering only the physically relevant part of the internal force vector (neglecting the contribution of the hourglass forces), by adjusting the peak cohesive traction $T_{0}$ to a value $T_{0}^{*}$, so that nodal equilibrium is restored and the spurious acceleration jump at the moment of node duplication is avoided. The equilibrium at the original corner node $i$ belonging to the $i$-th fiber, in the direction of the normal $\mathbf{n}_{i}$ to the crack flanks in the deformed configuration at the $i$-th corner node, writes as:

$$
\left(\mathbf{f}_{i n, i}^{m}+\mathbf{f}_{i n t, i}^{s, m}-\mathbf{f}_{e x t, i}^{m}\right) \cdot \mathbf{n}_{i}=-\left(\mathbf{f}_{c o h, i}^{m, *}+\mathbf{f}_{c o h, i}^{m, o l d}\right) \cdot \mathbf{n}_{i}
$$

where $\mathbf{f}_{c o h, i}^{m, \text { old }}$ is the contribution to the nodal cohesive forces due to the already existent cohesive elements, while $\mathbf{f}_{c o h, i}^{m, *}$ is the contribution of the newly inserted interface elements with the adjusted peak cohesive traction, defined as

$\mathbf{f}_{c o h, i}^{m, *}=\frac{\mathbf{F}_{c o h, i}^{u p, *}+\mathbf{F}_{c o h, i}^{l o w, *}}{2}$ with

$$
\begin{aligned}
& \mathbf{F}_{c o h, i}^{u p, *}=\mathcal{A}_{e=1}^{N_{i}^{u p}}\left(\sum_{k=1}^{N_{G P}} 2 w_{k} J_{\Gamma_{k}} \mathbf{T}_{0, k}^{*} N_{i k}^{u p}\right)^{e} \\
& \mathbf{F}_{c o h, i}^{\text {low }, *}=\mathcal{A}_{e=1}^{N_{i}^{l o w}}\left(\sum_{k=1}^{N_{G P}} 2 w_{k} J_{\Gamma_{k}} \mathbf{T}_{0, k}^{*} N_{i k}^{l o w}\right)^{e}
\end{aligned}
$$

where $\mathbf{T}_{0, k}^{*, e}=T_{0}^{*, e} \mathbf{n}_{k}^{e}$, being $T_{0}^{*, e}$ the unknown peak cohesive traction for the newly inserted interface element $e$, assumed to be equal at all Gauss points of the element. After computing the new peak value $T_{0}^{*, e}$ from eq. 24, the string critical length is rescaled to maintain the same fracture energy $\mathcal{G}_{c}$, i.e. $l_{c}^{*, e}=2 \mathcal{G}_{c} / T_{0}^{*, e}$. The modification of the maximum cohesive traction has been shown $[2,34,32,38]$ to not significantly alter the overall mechanical response, since the dissipative process is mainly governed by the fracture energy. All the DCEs of the same interface element are hence assigned the same adjusted cohesive properties.

Following the approach proposed in [18], additional fictitious nodal forces are then introduced to compensate only the part of the equivalent nodal internal forces due to the hourglass control. This second nodal correction is assumed to linearly decrease from its initial value to zero during a fixed number of time steps, determined on the basis of the size of the inserted interface element.

\section{Numerical examples}

Four numerical examples are considered in this section. The effect of the proposed correction procedure is assessed in the first two cases, where two fracture propagation problems are simulated. The last two examples concern the simulation of blade cutting problems to investigate the effect of increasing the number of DCEs per interface element to better describe the interaction between the blade and the process zone. All the tests are carried out in an explicit dynamics framework.

\subsection{Three point bending specimen}

A three point bending specimen $[7,9]$ is first considered (Figure 7) with dimensions $L=600 \mathrm{~mm}$ and $H=150$ $\mathrm{mm}$ in the vertical plane, and an out-of-plane thickness $B=5 \mathrm{~mm}$. Several discretizations are adopted, each one characterized by a different element size in the vertical direction $h_{e}=7.5,10,15,25,30 \mathrm{~mm}$, constant along the beam height, while the element width is always fixed to $10 \mathrm{~mm}$. The case $h_{e}=30 \mathrm{~mm}$ is depicted in Figure 7. The material properties, taken from [9], are the following: Young's modulus $E=36,500 \mathrm{MPa}$, 
Poisson's ratio $\nu=0.1$, cohesive strength $T_{0}=3.19$ $\mathrm{MPa}$ and mode I fracture toughness $\mathcal{G}_{c}=0.05 \mathrm{~N} / \mathrm{mm}$. The resulting cohesive length $\ell_{c}=E \mathcal{G}_{c} / T_{0}^{2}=179 \mathrm{~mm}$ is larger than the beam height, thus an appropriate, minimum number of integration points in the cohesive process zone, e.g. a minimum of 3-5 interface elements, is easily guaranteed for each of the five simulation cases. The simulation is carried out imposing that the downward vertical displacement $v(t)$ of the top middle point of the beam (see Figure 7) is linearly increasing with a constant velocity of $2 \mathrm{~mm} / \mathrm{s}$. A Rayleigh damping with coefficients $\alpha=10^{-3}$ (multiplying the mass matrix) and $\beta=1 \cdot 10^{-7}$ (multiplying the stiffness matrix) is introduced to approximate a quasi-static response and to limit dynamic oscillations. The Rayleigh damping coefficients have been selected through a sensitivity study so as to obtain a quasi-static response in the initial elastic phase, i.e. guaranteeing that the contribution of the inertia forces to the overall balance is small enough, without overdamping the structural response.

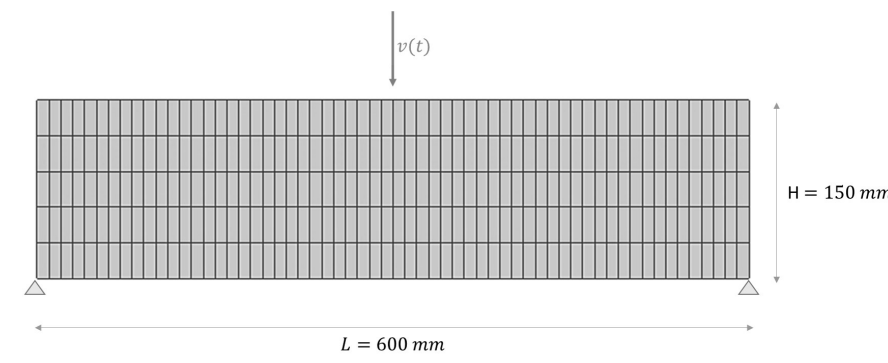

Fig. 7 Three point bending specimen, (coarser) mesh size $h_{e}=30 \mathrm{~mm}$.

The influence of the mesh size is assessed by fixing the number of DCEs per interface element to one, and by varying $h_{e}$. The comparison in terms of nondimensional load-middle point displacement and crackmouth acceleration-displacement curves is shown in Figure 8. Because of the displacement control, the (actual) snap-back behaviour results in a sudden load decrease, compensated by the growth of inertia forces (Figure 8b); after this drop, two different follow-up are observed: the simulations with mesh sizes $h_{e}=7.5,10,15$ show responses decreasing with continuity, while those with mesh sizes $h_{e}=25,30$ evidence a pathological behaviour in the post-peak regime. The choice of the space-time discretization influences the outcome also in terms of the acceleration behaviour. Focusing on the acceleration vs displacement plot, three distinct phases can be appreciated. In the first one, preceding the load drop, the first opening of the element faces and DCE insertion produce steep but isolated acceleration peaks, resulting in a progressive slope reduction on the loaddisplacement response; the second set of acceleration peaks corresponds to the load drop event, when the snap-back produces, despite damping, rather strong oscillations that tend to vanish only after a not negligible time interval; in the third set, observed in the post-peak regime, the acceleration increases due to the duplication of the last but one node along the ligament. It is worthwhile to emphasize that no disturbance is introduced by the vanishing DCEs, since the exchanged cohesive forces are almost zero when they approach their critical length and then disappear.
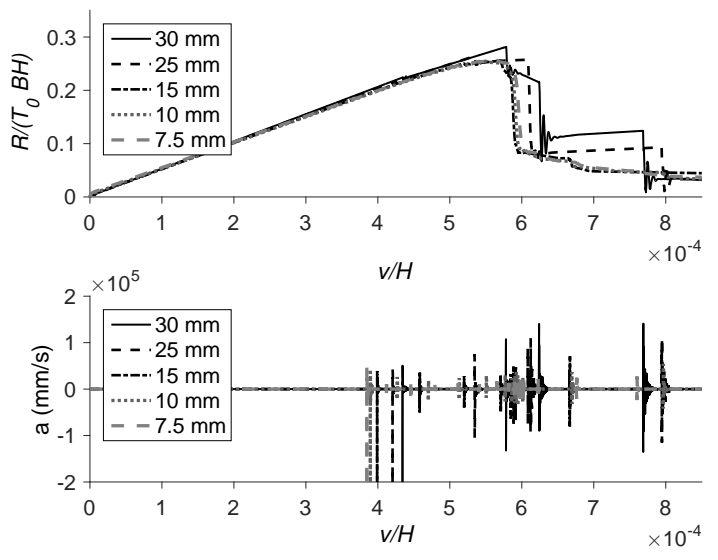

Fig. 8 Three point bending specimen. Comparison in terms of load-displacement response and acceleration at the crack mouth between different meshes (one DCE per element case).

The effectiveness of the correction procedure, discussed in section 2, is assessed in Figure 9, where the load-displacements curves, obtained with and without the correction, are compared for the cases $h_{e}=15$ $\mathrm{mm}$ and $h_{e}=30 \mathrm{~mm}$. The correction method produces smoother load-displacement curves, while the responses obtained without the correction method show non physical jumps, by-products of the spurious wave oscillations propagating because of the lack of nodal equilibrium. Table 2 reports the maximum of the elemental values of the corrected cohesive strengths $T_{0}^{*, \max }=$ $\max \left\{T_{0}^{*, e}\right\}$ for the five adopted discretizations. The ratio between the maximum adjusted cohesive strengths $T_{0}^{*, \max }$ and the initial cohesive strength $T_{0}$ in all the considered cases is between 1.28 and 1.75 times and decreases with decreasing element size.

The sensitivity of the load-displacement response with respect to the two Rayleigh damping parameters, in the case $h_{e}=15 \mathrm{~mm}$, is shown in Figures 10a and $10 \mathrm{~b}$, in which $\alpha$ and $\beta$ are varied once at a time. Reducing either the mass-related parameter $\alpha$ or the stiffness- 


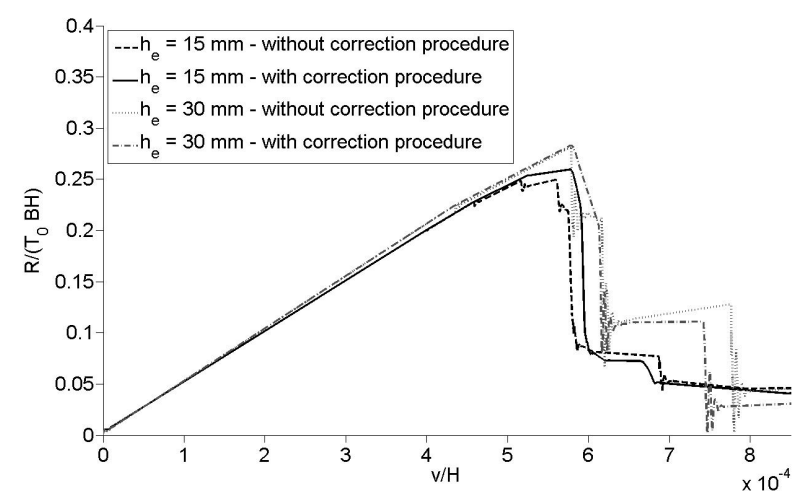

Fig. 9 Three point bending specimen. Effect of the correction procedure, $h_{e}=15-30 \mathrm{~mm}$.

Table 2 Three point bending specimen. Peak cohesive tractions after the correction procedure

\begin{tabular}{ccc}
\hline$h_{e}$ & $T_{0}^{*, \max }=\max _{e}\left\{T_{0}^{*, e}\right\}$ & $\frac{T_{0}^{*, \max }}{T_{0}}$ \\
\hline 7.5 & $4.08 \mathrm{MPa}$ & 1.28 \\
10 & $4.15 \mathrm{MPa}$ & 1.30 \\
15 & $4.34 \mathrm{MPa}$ & 1.36 \\
25 & $5.10 \mathrm{MPa}$ & 1.60 \\
30 & $5.58 \mathrm{MPa}$ & 1.75
\end{tabular}

related one $\beta$ by an order of magnitude produces a clear oscillatory behavior (gray solid lines) already in the initial part of the elastic response, while the selected parameters guarantee a quasi-static response. It should also be noted that the peak value and the post-peak behavior, except that for the reduced oscillations, are not significantly affected.

Finally, the comparison between the numerical results obtained with one and two DCEs per interface element is shown in Figure 11 for the intermediate mesh with $h_{e}=15 \mathrm{~mm}$, in combination with the correction method of section 2. The load drop is correctly reproduced; however, doubling the number of DCEs does not visibly improve the numerical result. This is due to the fact that the element size is far smaller than the cohesive length. In view of the linear edge kinematics, the configuration change at the crack tip depends only on the displacements of the nodes of the opening element edges, so that in this case no advantage comes from adding more cohesive strings between linearly opening faces

\subsection{Coarse regular mesh with a rectilinear crack}

The example considered in [19] to assess the effectiveness of their smoothing procedure in the case of brittle fracture propagation modeled with the X-FEM method
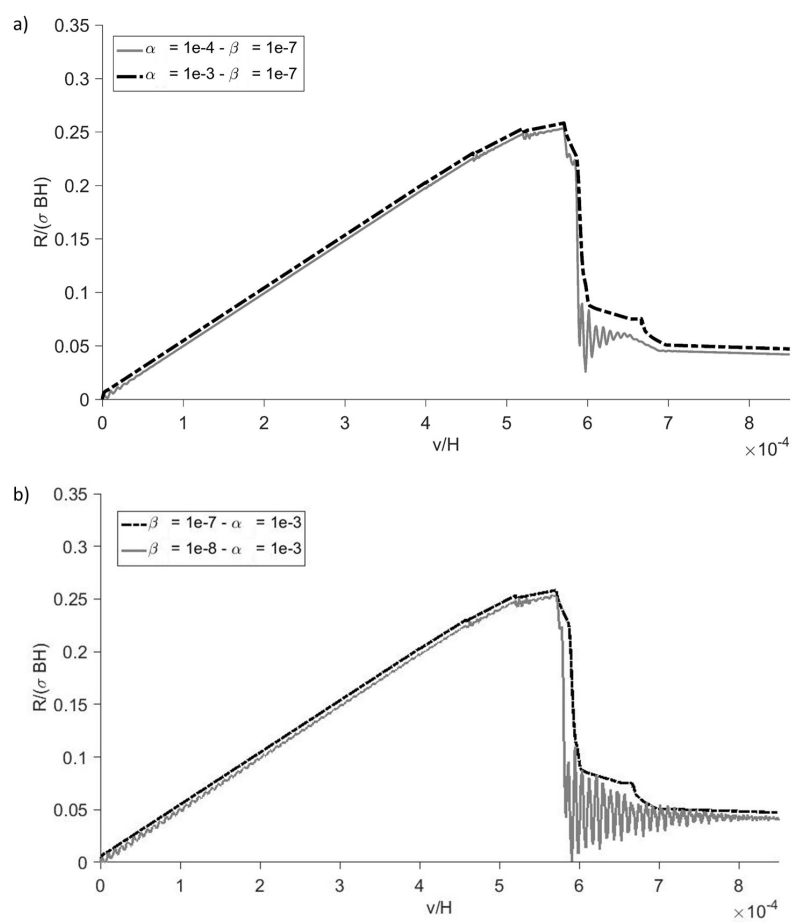

Fig. 10 Three point bending specimen. Sensitivity of structural response to Rayleigh damping parameters for $h_{e}=$ $15 \mathrm{~mm}$ : a) effect of reducing $\alpha$ for $\beta=1 \cdot 10^{-7}$; b) effect of reducing $\beta$ for $\alpha=1 \cdot 10^{-3}$.

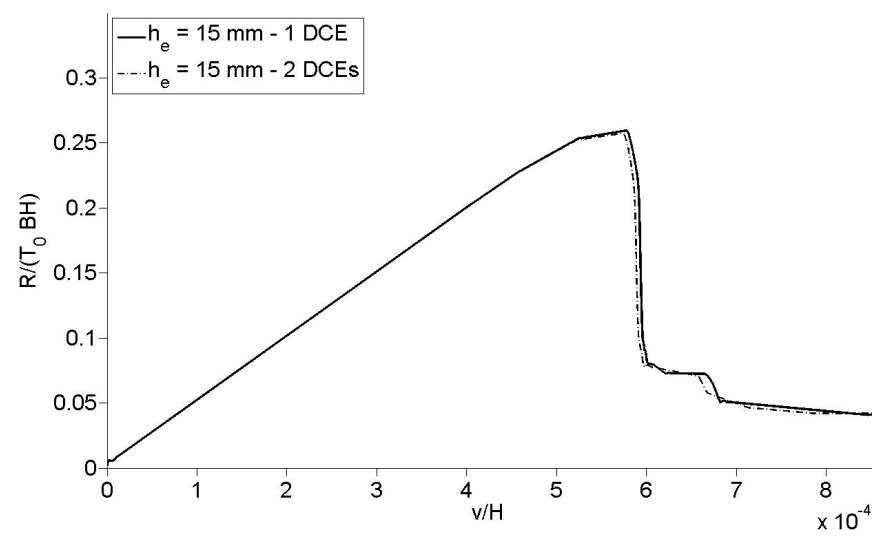

Fig. 11 Three point bending specimen. Comparison of load displacement curves for different number of DCEs, $h_{e}=15$ $\mathrm{mm}$.

is here reproduced and adapted to the case of cohesive behavior. A rectilinear crack is propagated in a rectangular domain discretized with the coarse and regular mesh shown in Figure 12a by prescribing an advancing crack tip velocity of $1 \mathrm{~m} / \mathrm{s}$. The bottom edge of the sample is fully constrained, while a uniform stress $\sigma=10 \mathrm{kPa}$ is applied at the top edge. Dimensions are the following: length $L=0.2 \mathrm{~m}$, height $H=0.1 \mathrm{~m}$, 
initial crack length $a_{i}=0.04 \mathrm{~m}$. The material is linear elastic, with Young's modulus $E=1 \mathrm{MPa}$, Poisson's ratio $\nu=0.3$, and mass density $\rho=1 \mathrm{~kg} / \mathrm{m}^{3}$. The reference cohesive strength is $T_{0}=10 \mathrm{kPa}$, to be adjusted with the correction procedure, while the fracture energy is $\mathcal{G}_{c}=0.05 \mathrm{~N} / \mathrm{mm}$. A Rayleigh damping with coefficients $\alpha=10^{-3}$ and $\beta=10^{-7}$ is adopted.

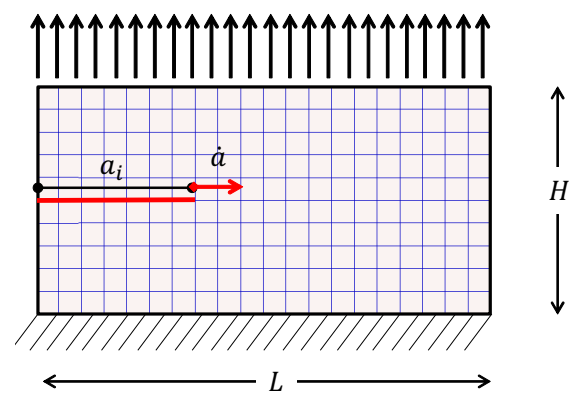

(a)

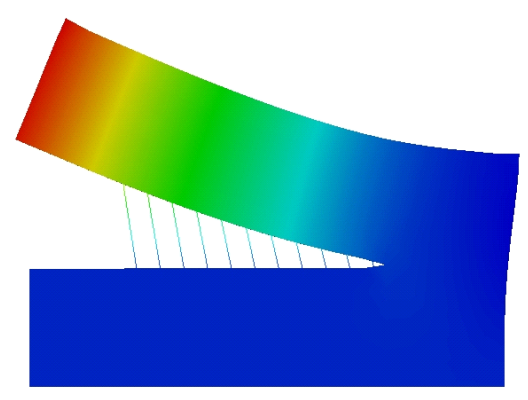

(b)

Fig. 12 Rectilinear crack propagation. (a) geometry, load and boundary conditions and (b) deformed shape.

The simulation is performed with and without the correction procedure described in section 2 and the vertical displacement and velocity of the top left corner node of the sample are recorded. The comparison between the two numerical results is shown in Figures 13a and $b$. The displacement vs time response is smoother when the nodal force correction is activated; it is instead strongly discontinuous without the correction. The effect of the correction becomes more evident as the time increases and the the crack propagates rightward. Moreover, the correction procedure also decreases the peaks and reduces the oscillations in the velocity response. As pointed out in [19], the velocity of the top left corner point obtained without any correction is completely not physical, while its values turn out to be more realistic when the correction is applied.

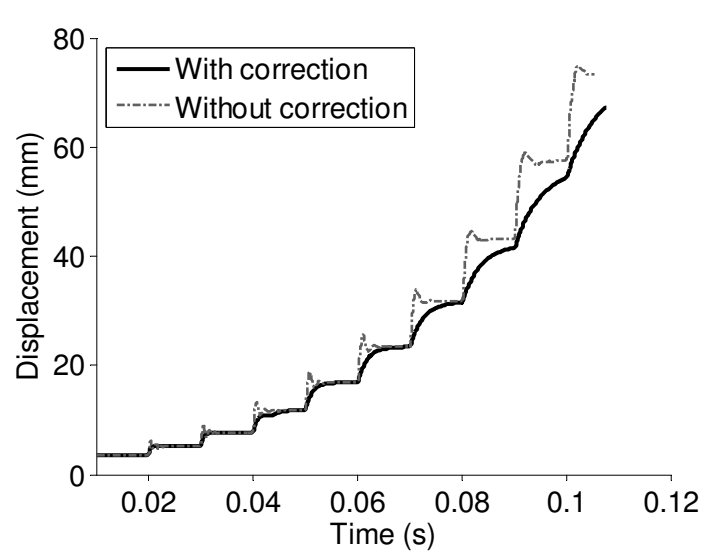

(a)

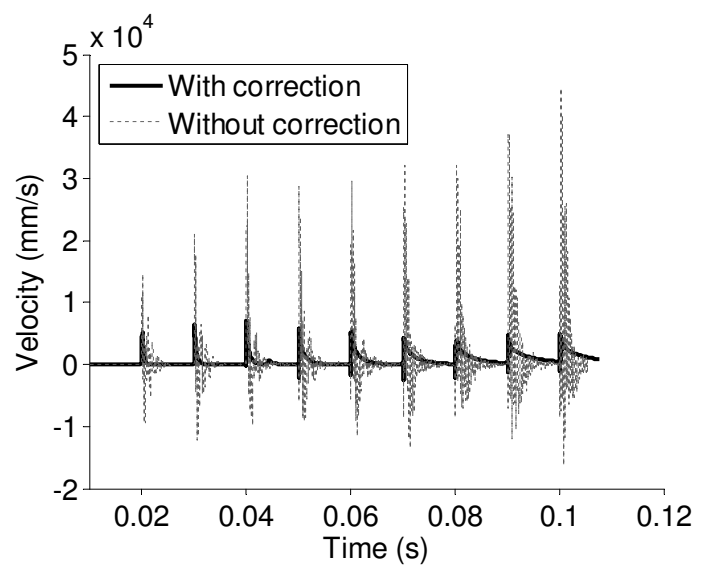

(b)

Fig. 13 Rectilinear crack propagation. Comparisons between corrected and not corrected responses in terms of (a) vertical displacement and (b) velocity at the top left corner.

\subsection{Cutting of a steel plate by a wedge}

The simulation of the cutting of a steel plate by a wedge, as in the experiments performed by $\mathrm{Lu}$ and Calladine [16], is considered. The plate is kept slightly inclined w.r.t. the horizontal plane, as shown in our model, depicted in Figure 14, where $\alpha=10^{\circ}$ is assumed. Nominal dimensions of the specimen are also included in the same picture. A pre-cut has been introduced into the model to facilitate the cutting initiation by the rigid wedge, which is made to advance steadily in a rectilinear direction along the horizontal plane. An elastic, perfectly plastic constitutive behavior has been assumed for the steel, with the following material parameters: Young's modulus $E=210 \mathrm{GPa}$, Poisson's ratio $\nu=0.3$, yield stress $\sigma_{y}=272 \mathrm{MPa}$, density $\rho=7,800 \mathrm{~kg} / \mathrm{m}^{3}$, fracture energy $\mathcal{G}_{c}=353$ $\mathrm{kJ} / \mathrm{m}^{2}$. A critical equivalent plastic strain $\varepsilon^{p}=0.15$ is considered for the activation criterion, while a lin- 

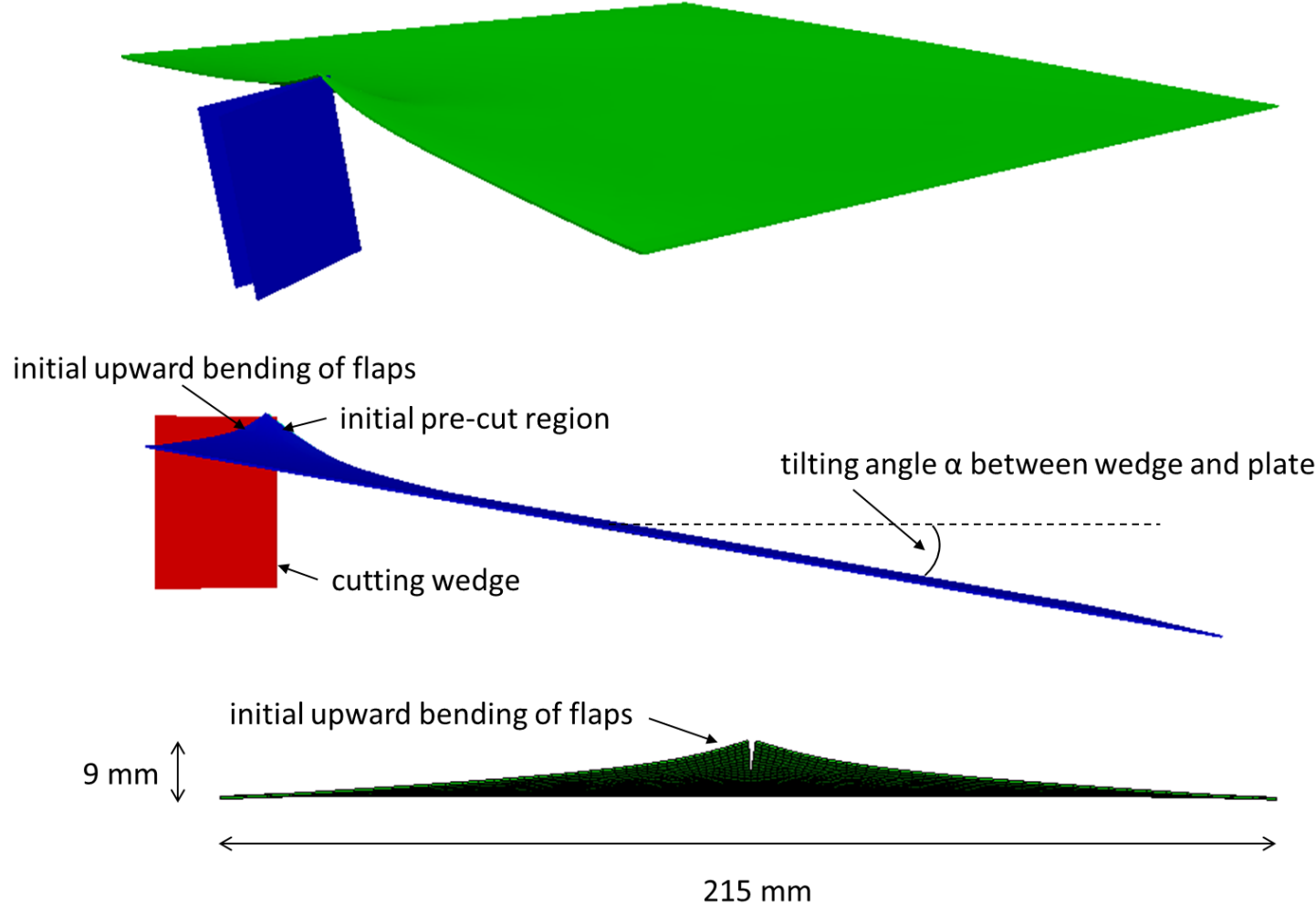

Fig. 14 Cutting of a steel plate by a wedge. Isometric and lateral views of square-shaped steel plate and wedge (from experiment in [16]).

ear softening law, with peak cohesive traction $T_{0}=408$ MPa, describes the cohesive behavior. The peak value $T_{0}$ is set equal to 1.5 times the yield stress $\sigma_{y}$, so that a plastic zone can develop ahead of the crack tip. The effect on crack propagation of the ratio $T_{0} / \sigma_{y}$ has been systematically studied by Tvergaard and Hutchinson (1992) [35] for ductile materials in plane strain. They have shown how this ratio should not be excessively low to allow for a complete formation of the plastic zone ahead of the crack tip. In the present case of a plate made of a ductile material, the criterion used for the activation of node separation is based on the equivalent plastic strain accumulated in the bulk, so that cohesive crack propagation is initiated when the prescribed threshold on the equivalent plastic strain is exceeded, irrespective of the cohesive strength. On the other hand, solid-shell finite elements, with only one solid-shell element and five integration points through the thickness, have been used for the plate discretization. The stress state at the Gauss points is three-dimensional, with a small, but non-zero normal stress in the thickness direction, so that plane stress conditions cannot be exactly assumed. As in Tvergaard and Hutchinson (1992), preliminary tests have been conducted with different $T_{0} / \sigma_{y}$ ratios and good results have been obtained with a value of 1.5. The typical in-plane size of the finite elements in the cutting region is $1 \mathrm{~mm}$, which has to be compared to a cohesive process zone characteristic length $\ell_{c}=E \mathcal{G}_{c} / T_{0}^{2}=1,002 \mathrm{~mm}$, longer than the plate in-plane size. A Rayleigh damping with coefficients $\alpha=10^{-3}$ and $\beta=10^{-8}$ is adopted.

Contours of the equivalent plastic strain are shown on the deformed configuration in Figure 15 at different steps of the wedge advancement.

Experimental data include friction between the wedge and the plate, while this is not taken into account in the numerical simulation. For this reason, the outcome of the numerical solution is compared with the analytical formula, based on a kinematic approach, proposed by Simonsen and Wierzbicki (1997) in [29] for estimating an upper bound to the cutting force $F_{a n}$, in which the frictional contribution is also neglected. Figure 16 shows the numerical non-dimensional forcedisplacement curves and the analytical estimate $F_{a n}=$ $623 \mathrm{~N}$ (horizontal red line). After an initial transition with a steady increase, the three numerical solutions, computed with a different number of DCEs per element face, oscillate around the analytical estimate, with no noticeable improvement brought by the increased number of DCEs. This can be explained by noting that in 


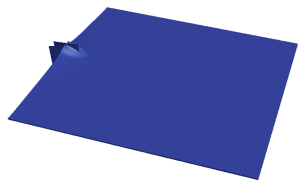

(a)

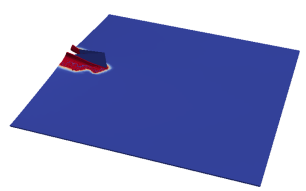

(c)

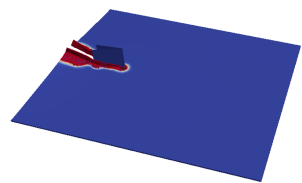

(e)

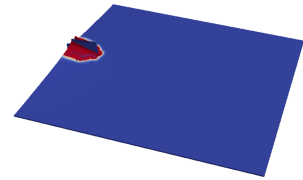

(b)

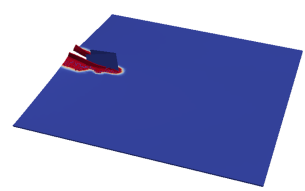

(d)

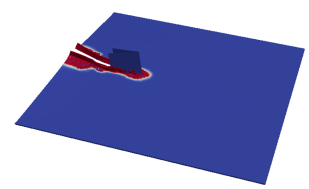

(f)
Fig. 15 Cutting of a steel plate by a wedge. Equivalent plastic strain contours at different stages of wedge advancement: a) $\mathrm{U}=8 \mathrm{~mm}, \mathrm{~b}) \mathrm{U}=20 \mathrm{~mm}, \mathrm{c}) \mathrm{U}=32 \mathrm{~mm}, \mathrm{~d}) \mathrm{U}=40 \mathrm{~mm}, \mathrm{e})$ $\mathrm{U}=48 \mathrm{~mm}, \mathrm{f}) \mathrm{U}=60 \mathrm{~mm}$, being $\mathrm{U}$ the blade displacement.

this case the reaction force on the wedge is determined to a large extent by the contact against the plastically deforming sides of the cut. The forces due to the deformation of the process zone play only a minor role, making it difficult to notice the effect of the number of DCEs. The strong oscillatory behavior of the numerical solution is mainly due to the dynamic contact between the blade and the progressively opening crack faces that induces high frequency stress waves. This dynamic effect, which is not considered in the analytical solution of Simonsen and Wierzbicki [29], could be partially mitigated adopting a finer spatial discretization and reducing the advancement speed of the blade, at the cost, however, of a significant increase of the computational burden.

\subsection{Thin plate cut by scissors}

As a final example, the simulation of the cutting process of a thin plate by a pair of scissors is considered. This problem is of particular interest in the field of virtual and/or robotized surgery, since obtaining a physically-based, reliable haptic feedback is mandatory to provide an effective tool for medical training and for robotized, remotely controlled interventions. The experimental setup proposed in [17] to characterize the scissoring force necessary to cut samples of different materials is here considered. Two instrumented Metzenbaum scissors blades rotate around their pivot under the action of a robot arm and a rectangular specimen, kept fixed at the two edges parallel to the cut direction, is progressively cut along its medial axis (see Figure 17). The pivot position remains fixed during the experiment. A force sensor measures the force $F$ applied to the scissors arm in a direction normal to the arm, so that the applied torque is linearly proportional to the force. The arm rotation angle $\theta$ is obtained by monitoring the angular velocity. The distance between the pivot and the force sensor is equal to $98 \mathrm{~mm}$.

Among the different materials cut in the experiments reported in [17], the case of chicken skin samples, of slightly different and irregular thicknesses, is here considered. The dimensions of the specimen are: $L=100 \mathrm{~mm}, B=23 \mathrm{~mm}, t=1.0 \mathrm{~mm}$ (see Figure 18 ); the thickness $t$ used for the simulation is the average of the nominal thicknesses of the three samples tested by the authors. The material properties are the following: Young's modulus $E=8 \mathrm{GPa}$, Poisson's ratio $\nu=0.4$, cohesive strength $T_{0}=0.2 \mathrm{MPa}$, density $\rho=1,800 \mathrm{~kg} / \mathrm{m}^{3}$, fracture energy $\mathcal{G}_{c}=2.8 \mathrm{~kJ} / \mathrm{m}^{2}$. Three different simulations are carried out considering an increasing number of DCE per element face, namely 1,2 and 3.

Taking into account the symmetry with respect to the middle surface of the specimen, only the upper half of the plate and a single blade of the scissors are modeled, as shown in Figure 18. Hence, the out-of-plane displacements of the nodes belonging to the symmetry plane are fully restrained. A rigid rotation around the pivot, with $\theta$ increasing in time with a constant angular velocity of 300 degrees/s (i.e. 100 times the experimental value), is imposed to the blade. A Rayleigh damping with coefficients $\alpha=10^{-3}$ and $\beta=10^{-8}$ is adopted to reduce the oscillations in the mechanical response.

A finite element mesh (see Figure 19) with only one solid-shell element through the thickness is used. The typical in-plane size of the finite elements in the cutting region is $0.5 \mathrm{~mm}$, which has to be compared to a cohesive process zone characteristic length $\ell_{c}=G \mathcal{G}_{c} / T_{0}^{2}=$ $2 \cdot 10^{5} \mathrm{~mm}$ ( $G$ being the shear modulus), significantly longer than the sample width.

Since only a single blade is considered, it is necessary to adapt the DCE concept to the particular case of the scissoring process, in which the mechanical response is governed by the interaction between the two scissors blades. As explained in section 2.2, when the contact between a single DCE and the blade is detected, the 


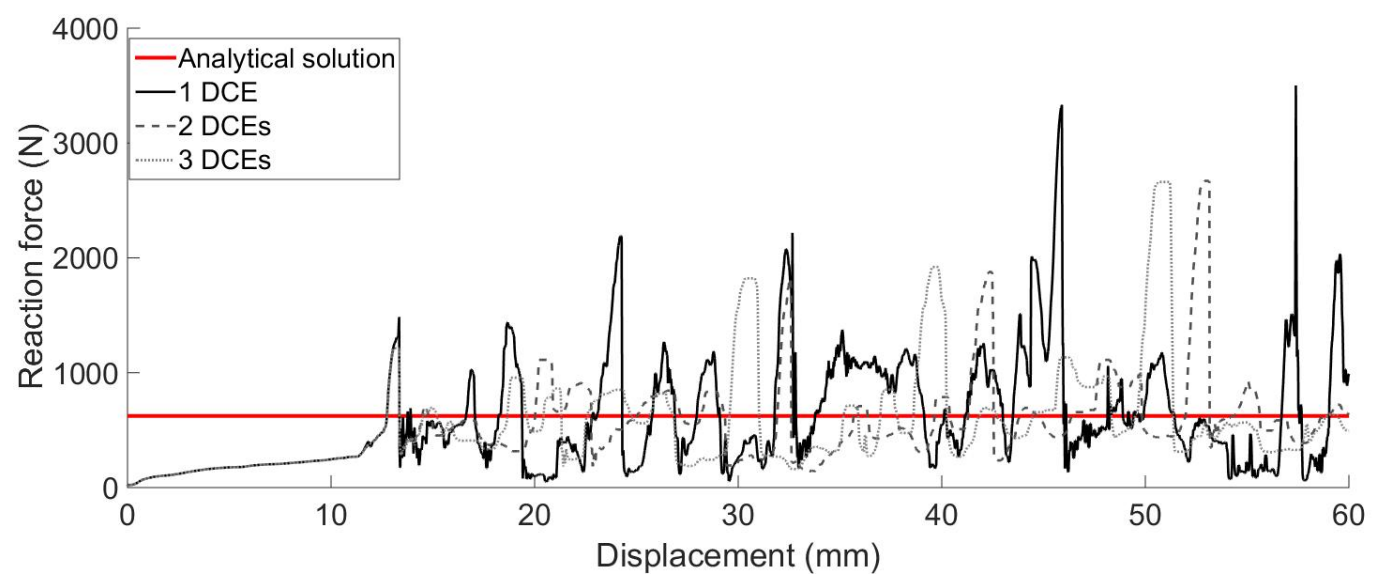

Fig. 16 Cutting of a steel plate by a wedge. Non-dimensional reaction force vs wedge displacement. Numerical solutions with increasing number of DCEs per element face. Horizontal solid line denotes analytical estimate.

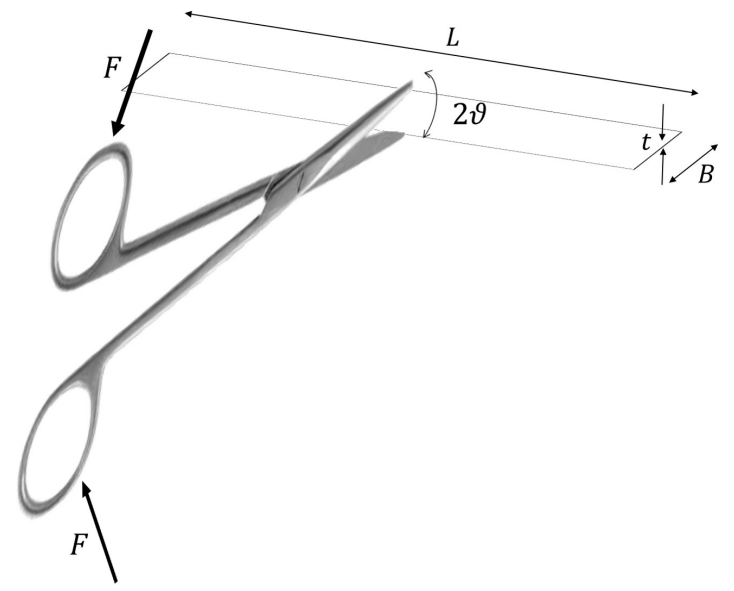

Fig. 17 Thin plate cut by scissors. Problem definition.

string is split into two branches. During its rigid rotation, the blade drags the contact point $\mathbf{x}^{c}$ downwards (if the upper part of the specimen is considered). It is assumed that the two cutting blades of the scissors meet when the contact point crosses the symmetry plane of the specimen. When this condition is met, contact with the second blade is accounted for by considering a third branch in the string, as shown in Figure 20, which connects the contact point $\mathbf{x}^{c}$ to its symmetrical $\mathbf{x}^{\prime c}$ on the other side of the symmetry plane. The directions of the cohesive forces transmitted to the crack flanks are modified according to the position of both $\mathbf{x}^{c}$ and $\mathbf{x}^{\prime c}$, so that the action of the two blades is accounted for. Moreover, the string length is given by the sum of the lengths of the three branches, providing a higher dissipation when the two blades are overlapping.

The force recorded during the experiment includes both the force necessary to cut the sample and the

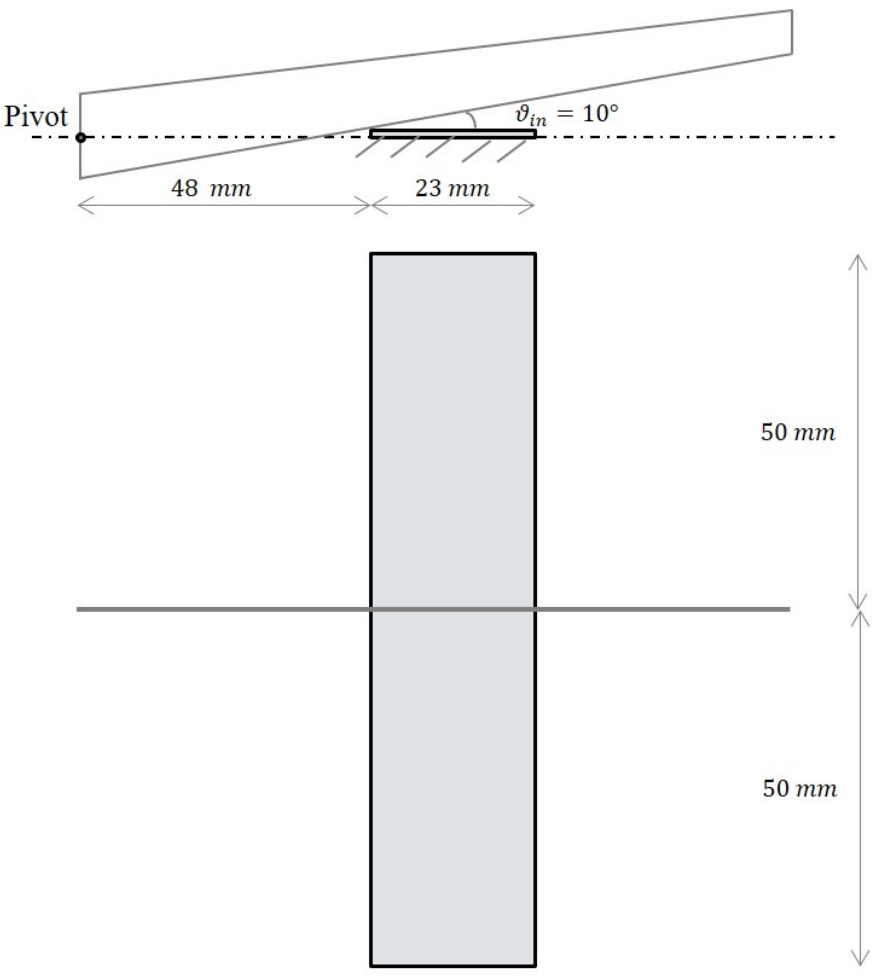

Fig. 18 Thin plate cut by scissors. Adopted model.

friction. The adopted numerical model is not able to simulate the frictional contribution. Anyway, the authors provide the force vs rotation curve of the scissors alone (Figure 21). As pointed out in [6], the cutting force recorded by closing the scissors over the air can be assumed to be representative of the overall frictional contribution, since the friction is mainly due to the interaction between the two blades. In comparing the outcome of the simulations to the experimental response, 


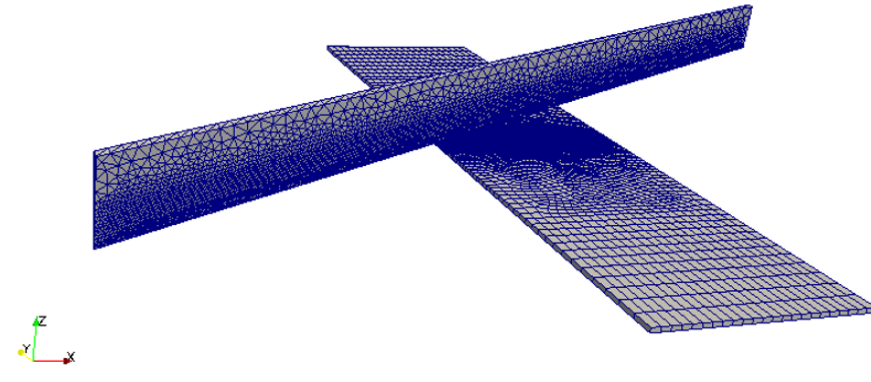

Fig. 19 Thin plate cut by scissors. Adopted discretization.

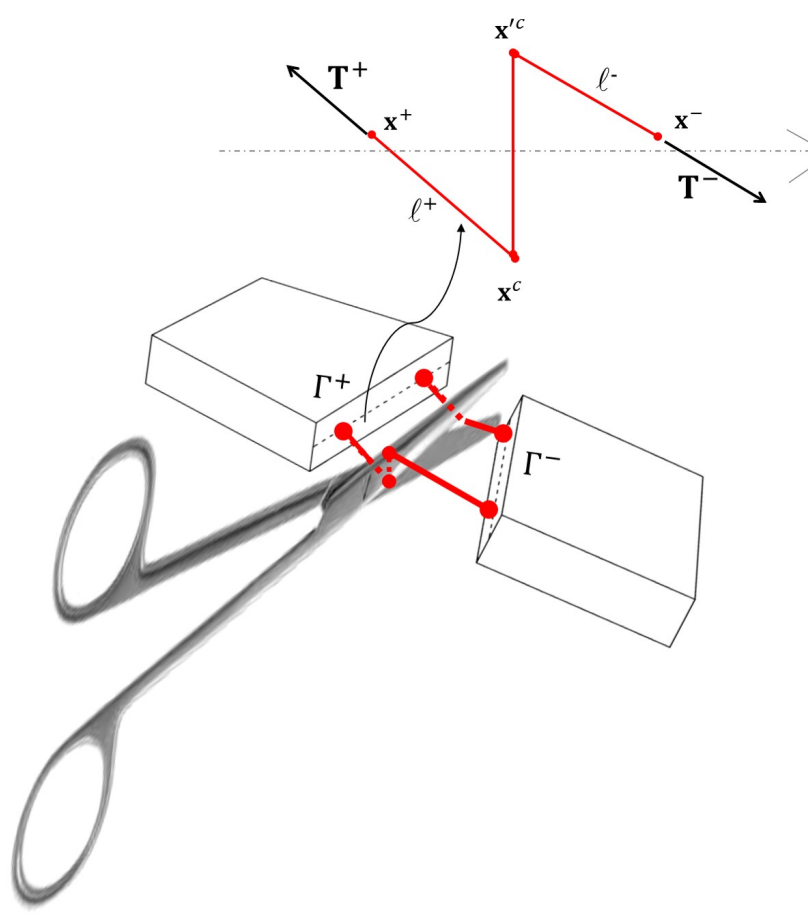

Fig. 20 Thin plate cut by scissors. Directional cohesive elements in case of scissor blades interaction.

the provided friction force, depicted in Figure 21 as a function of the angle, is then added to the resulting numerical cutting force.

Figure 22 shows the comparison between the simulation and the experimental results. The overall cutting force is determined by imposing the rotational equilibrium around the scissors pivot of all the forces acting on the blade. These are the cohesive contributions coming from all the DCEs for which contact with the blade has been detected and the contact forces between the rigid body and the solid-shell elements.

The angle shown in Figure 22 is the relative angle between the blades, i.e. twice the absolute angle between the blade and the horizontal axis; therefore, the cutting process starts at about $20^{\circ}$ and decreases to $0^{\circ}$

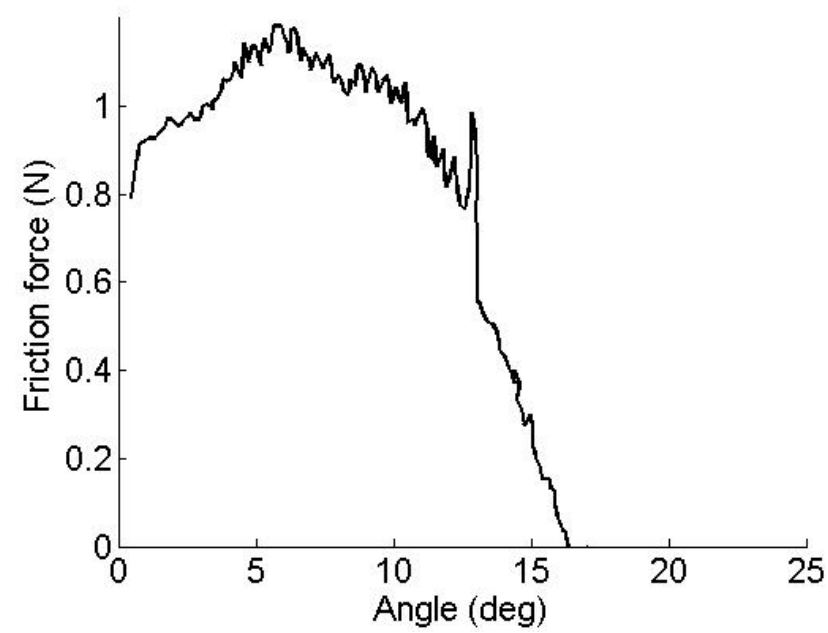

Fig. 21 Thin plate cut by scissors. Frictional force between scissors blades.

as the blade rotates towards the plate. In other words, the curves are walked through from right to left, as in the original work [17]. Following the numbering sequence in Figure 22, from (circled) points 0 to 1 the blade is initially rotating rigidly, with no contact with the plate; from point 1 to point 2 the blade starts its interaction by deforming but still not cutting the plate; from point 2 to point 3 the actual cut occurs with severe oscillations in the response; finally, from point 3 to point 4 the plate has been completely cut by the blades. Despite a rather coarse mesh, the numerical simulation captures the deformation phase 1-2 and the cutting phase 2-3 reasonably well. The entity of the oscillations in the experimental curve during the latter phase 2-3 of the cut depends also on local effects (such as thickness variation), which are also most likely responsible for the dispersion in the experimental results shown by the three measured curves reproduced in Figure 22. Unlike in the work in [17], where the force rapidly drops at the end, some fluctuations in the numerical results can be observed in the phase $3-4$, due to the DCEs not yet completely cut during the last instants of the cutting process.

It should be noticed that the accuracy of the numerical results improves as the number of DCEs per element increases. The simulation carried out with only one DCE per element underestimates the initial stiffness during phase 1-2 and anticipates the final phase w.r.t. the 2-DCEs and 3 DCEs cases. The 1-DCE case also underestimates the plateau-like (with fluctuations) behavior during the phase 2-3, which instead is better reproduced by the 2-DCEs and 3-DCEs simulations. The differences between the 2-DCEs and 3-DCEs cases 
are rather small: the addition of one more cable increases slightly the entity of fluctuations during the first part of the cut and it also affects the last phase $3-4$, because of the higher number of still interacting strings.

The energy dissipated (proportional to the area underneath the force-rotation curve) by the simulations with 2 and 3-DCEs compares well to the energy dissipated in two of the experimental tests, while it is significantly smaller than in the third test that exhibits an anomalous peak force.

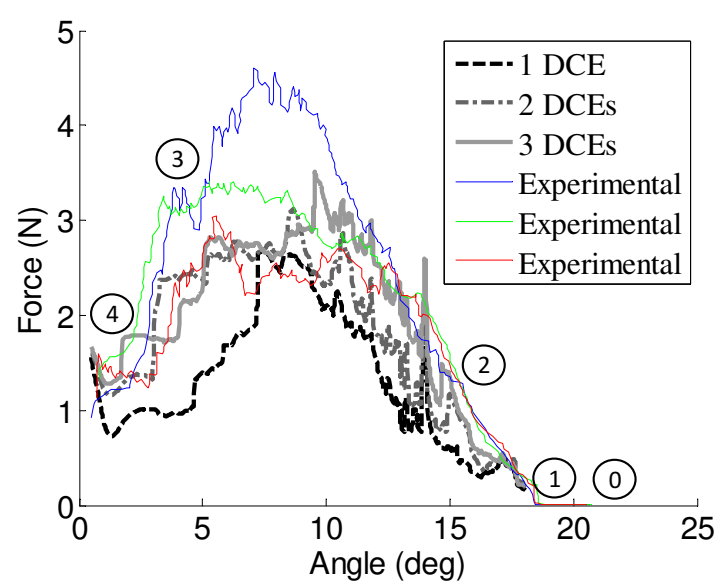

Fig. 22 Thin plate cut by scissors. Cutting of a chicken skin specimen: cutting force vs rotation angle. Comparison of experimental and numerical response for increasing number of DCEs. Experimental curves refer to specimens of nonuniform, irregular thicknesses.

\section{Conclusions}

This work is focused on the discussion of some computational issues concerning the simulation of blade cutting of thin walled structures. The adopted numerical strategy is based on the use of the directional cohesive elements proposed in $[11,22]$. These elements allow to resolve the scale of the blade radius without adopting an extremely refined discretization. The concept of directional cohesive elements has here been extended for application to the cutting by scissors, accounting for the interaction within the blade pair and the effect of an increased number of directional cohesive elements per opening face has been elucidated.

The second aspect investigated in this contribution is the presence of unphysical acceleration jumps in the numerical response due to the lack of nodal equilibrium at the moment of node duplication and insertion of an interface element. The unbalanced nodal forces induce the propagation of spurious stress waves than can lead to significant deviations in the local stress field and affect the accuracy of the simulation. A correction procedure, based on the ri-definition of the maximum cohesive traction in order to guarantee the nodal equilibrium is proposed. The computational techniques studied in this work have been shown to play a beneficial role in the development of a robust numerical tool, able to simulate the complex mechanical behavior involved in the cutting process.

\section{Acknowledgements}

The financial support by Tetra Pak Packaging Solutions is kindly acknowledged. Moreover, we acknowledge the CINECA and the Regione Lombardia award under the LISA initiative 2016-2018, for the availability of high performance computing resources and support.

\section{Compliance with Ethical Standards:}

Funding: This study was partly funded by Tetra Pak Packaging Solutions Spa (Order n. 800120646, 2016, Company code 0102) and partly by the CINECA and the Regione Lombardia award under the LISA initiative 2016-2018.

Conflict of Interest: Author Umberto Perego has received a research grant from Company Tetra Pak Packaging Solutions Spa. The remaining authors declare that they have no conflict of interest.

\section{References}

1. Alfano, G.: On the influence of the shape of the interface law on the application of cohesive-zone models. Composites Science and Technology 66(6), 723-730 (2006). DOI 10.1016/j.compscitech.2004.12.024

2. Alfano, G., Crisfield, M.A.: Finite element interface models for the delamination analysis of laminate composites: mechancial and computational issues. International Journal for Numerical Methods in Engineering 50, 1701-1736 (2001). DOI 10.1002/nme.93

3. Ambati, M., De Lorenzis, L.: Phase-field modeling of brittle and ductile fracture in shells with isogeometric NURBS-based solid-shell elements. Computer Methods in Applied Mechanics and Engineering (2016). DOI 10.1016/j.cma.2016.02.017

4. Amiri, F., Millán, D., Shen, Y., Rabczuk, T., Arroyo, M.: Phase-field modeling of fracture in linear thin shells. Theoretical and Applied Fracture Mechanics 69, 102-109 (2014). DOI 10.1016/j.tafmec.2013.12.002

5. Areias, P.M.A., Belytschko, T.: Non-linear analysis of shells with arbitrary evolving cracks using XFEM. International Journal for Numerical Methods in Engineering 62(3), 384-415 (2005). DOI 10.1002/nme.1192

6. Atkins, A.G., Mai, Y.W.: On the guillotining of materials. Journal of Materials Science 14(11), 2747-2754 (1979). DOI 10.1007/bf00610649 
7. Carpinteri, A., Colombo, G.: Numerical analysis of catastrophic softening behaviour (snap-back instability). Computers \& Structures 31, 607-636 (1989). DOI 10.1016/0045-7949(89)90337-4

8. Cirak, F., Ortiz, M., Pandolfi, A.: A cohesive approach to thin-shell fracture and fragmentation. Computer Methods in Applied Mechanics and Engineering 194, 26042618 (2005). DOI 10.1016/j.cma.2004.07.048

9. Do, B., Liu, W., Yang, Q., Su, X.: Improved cohesive stress integration schemes for cohesive zone elements. Engineering Fracture Mechanics 107, $14-28$ (2013). DOI 10.1016/j.engfracmech.2013.04.009

10. Dolbow, J., Moës, N., Belytschko, T.: Modeling fracture in MindlinReissner plates with the extended finite element method. International Journal of Solids and Structures 37(48-50), 7161-7183 (2000). DOI 10.1016/s00207683(00)00194-3

11. Frangi, A., Pagani, M., Perego, U., Borsari, R.: Directional Cohesive Elements for the Simulation of Blade Cutting of Thin Shells. Computer Modeling in Engineering \& Sciences 57, 205-224 (2010). DOI 10.3970/cmes.2010.057.205

12. Ghisi, A., Confalonieri, F., Perego, U.: Blade cutting simulation with crack propagation through thin-walled structures via solid-shell finite elements in explicit dynamics. In: PANACM 2015 - 1st Pan-American Congress on Computational Mechanics, in conjunction with the 11th Argentine Congress on Computational Mechanics, MECOM 2015, pp. 304-313 (2015)

13. Gilormini, P., Diani, J.: Testing some implementations of a cohesive-zone model at finite strain. Engineering Fracture Mechanics 148, 97-109 (2015). DOI 10.1016/j.engfracmech.2015.09.013

14. Harper, P.W., Hallett, S.R.: Cohesive zone length in numerical simulations of composite delamination. Engineering Fracture Mechanics 75(16), 4774-4792 (2008). DOI 10.1016/j.engfracmech.2008.06.004

15. Hu, N., Zemba, Y., Okabe, T., Yan, C., Fukunaga, H., Elmarakbi, A.M.: A new cohesive model for simulating delamination propagation in composite laminates under transverse loads. Mechanics of Materials 40(11), 920-935 (2008). DOI 10.1016/j.mechmat.2008.05.003

16. Lu, G., Calladine, C.: On the cutting of a plate by a wedge. International Journal of Mechanical Sciences 32(4), 293-313 (1990). DOI 10.1016/00207403(90)90095-Z

17. Mahvash, M., Voo, L., Kim, D., Jeung, K., Wainer, J., Okamura, A.M.: Modeling the forces of cutting with scissors. IEEE Trans Biomed Eng 55(3), 848-856 (2008). DOI 10.1109/TBME.2007.908069

18. Menouillard, T., Belytschko, T.: Correction force for releasing crack tip element with xfem and only discontinuous enrichment. European Journal of Computational Mechanics 18(5-6), 465-483 (2009). DOI 10.3166/ejcm.18.465-483

19. Menouillard, T., Belytschko, T.: Smoothed nodal forces for improved dynamic crack propagation modeling in XFEM. International Journal for Numerical Methods in Engineering 84, 47-72 (2010). DOI 10.1002/nme.2882

20. Mostofizadeh, S., Fagerström, M., Larsson, R.: Dynamic crack propagation in elastoplastic thin-walled structures: Modelling and validation. International Journal for $\mathrm{Nu}-$ mercal Methods in Engineering 96(2), 63-86 (2013). DOI 10.1002/nme.4524

21. Needleman, A.: Some issues in cohesive surface modeling. Procedia IUTAM 10, 221-246 (2014). DOI 10.1016/j.piutam.2014.01.020
22. Pagani, M., Perego, U.: Explicit dynamics simulation of blade cutting of thin elastoplastic shells using a "directional" cohesive elements in solid-shell finite element models. Computer Methods in Applied Mechanics and Engineering 285, 515-541 (2015). DOI 10.1016/j.cma.2014.11.027

23. Pandolfi, A., Ortiz, M.: An eigenerosion approach to brittle fracture. International Journal for Numerical Methods in Engineering 92(8), 694-714 (2012). DOI $10.1002 /$ nme. 4352

24. Planas, J., Elices, M.: Nonlinear fracture of cohesive materials. In: Z.P. Bažant (ed.) Current Trends in Concrete Fracture Research, pp. 139-157. Springer Netherlands (1991). DOI 10.1007/978-94-011-3638-9_10

25. Qiao, H., Chen, W.Q., Yang, Q., Lua, J.: Augmented Cohesive Elements for Efficient Delamination Analyses of Composite Laminates. Journal of Engineering Materials and Technology 133, 041,010-1 (2011). DOI $10.1115 / 1.4004694$

26. Schellekens, J.C.J., De Borst, R.: On the numerical integration of interface elements. International Journal for Numerical Methods in Engineering 36, 43-66 (1993). DOI 10.1002/nme.1620360104

27. Schwarze, M., Reese, S.: A reduced integration solid-shell finite element based on the eas and the ans concept: large deformation problems. International Journal for Numerical Methods in Engineering 85(3), 289-329 (2011). DOI $10.1002 /$ nme. 2966

28. Shet, C., Chandra, N.: Effect of the shape of t cohesive zone curves on the fracture response. Mechanics of Advanced Materials and Structures 11(3), 249-275 (2004). DOI 10.1080/15376490490427207

29. Simonsen, B.C., Wierzbicki, T.: Plasticity, fracture and friction in steady state plate cutting. International Journal of Impact Engineering 19(8), 667-691 (1997)

30. Smith, E.: The effect of the Stress-Relative displacement law on failure predictions using the cohesive zone model. International Journal of Fracture 99(1-2), 41-51 (1999). DOI 10.1023/a:1018320714262

31. Soto, A., González, E.V., Maimí, P., Turon, A., Sainz de Aja, J.R., de la Escalera, F.M.: Cohesive zone length of orthotropic materials undergoing delamination. Engineering Fracture Mechanics 159, 174-188 (2016). DOI 10.1016/j.engfracmech.2016.03.033

32. Turon, A., Camanho, P.P., Costa, J., Renart, J.: Accurate simulation of delamination growth under mixedmode loading using cohesive elements: Definition of interlaminar strengths and elastic stiffness. Composite Structures 92(8), 1857-1864 (2010). DOI 10.1016/j.compstruct.2010.01.012

33. Turon, A., Costa, J., Camanho, P., Maimí, P.: Analytical and numerical investigation of the length of the cohesive zone in delaminated composite materials. In: Mechanical Response of Composites, Computational Methods in Applied Sciences, vol. 10, pp. 77-97. Springer Netherlands (2008). DOI 10.1007/978-1-4020-8584-0_4

34. Turon, A., Dávila, C.G., Camanho, P.P., Costa, J.: An engineering solution for mesh size effects in the simulation of delamination using cohesive zone models. Engineering Fracture Mechanics 74(10), 1665-1682 (2007). DOI 10.1016/j.engfracmech.2006.08.025

35. Tvergaard, V., Hutchinson, J.W.: The relation between crack growth resistance and fracture process parameters in elastic-plastic solids. Journal of the Mechanics and Physics of Solids 40(6), 1377-1397 (1992). DOI 10.1016/0022-5096(92)90020-3 
36. Ulmer, H., Hofacker, M., Miehe, C.: Phase field modeling of fracture in plates and shells. PAMM Proceedings in Applied Mathematics and Mechanics 12(1), 171-172 (2012). DOI 10.1002/pamm.201210076

37. Volokh, K.Y.: Comparison between cohesive zone models. Communications in Numerical Methods and Engineering 20(11), 845-856 (2004). DOI 10.1002/cnm.717

38. Yang, Q., Cox, B.: Cohesive models for damage evolution in laminated composites. International Journal of Fracture 133(2), 107-137 (2005). DOI 10.1007/s10704005-4729-6

39. Yang, Q.D., Fang, X.J., Shi, J.X., Lua, J.: An improved cohesive element for shell delamination analyses. International Journal for Numerical Methods in Engineering 83(5), 611-641 (2010). DOI 10.1002/nme.2848 